\title{
Mesoscopic Charge Relaxation
}

\author{
Simon E. Nigg ${ }^{1}$, Rosa López ${ }^{2}$ and Markus Büttiker ${ }^{1}$ \\ ${ }^{1}$ Département de Physique Théorique, Université de Genève, CH-1211 Genève 4, Switzerland and \\ ${ }^{2}$ Departament de Física, Universitat de les Illes Balears, E-07122 Palma de Mallorca, Spain
}

(Dated: September 11, 2018)

\begin{abstract}
We consider charge relaxation in the mesoscopic equivalent of an RC circuit. For a single-channel, spin-polarized contact, self-consistent scattering theory predicts a universal charge relaxation resistance equal to half a resistance quantum independent of the transmission properties of the contact. This prediction is in good agreement with recent experimental results. We use a tunneling Hamiltonian formalism and show in Hartree-Fock approximation, that at zero temperature the charge relaxation resistance is universal even in the presence of Coulomb blockade effects. We explore departures from universality as a function of temperature and magnetic field.
\end{abstract}

PACS numbers: 85.35.Gv, 73.23.Hk, 73.21.La, 73.22.Dj

There is increasing interest in the dynamics of mesoscopic structures motivated by the desire to manipulate and measure quantum phenomena as rapidly as possible. It is thus of great importance to characterize the time scales governing the electron dynamics in simple mesoscopic structures. An elementary but fundamental building block is the quantum coherent capacitor [1]. As in the classical case, the low frequency dynamics of a mesoscopic capacitor is determined by a charge relaxation time $\tau_{R C}$. For a quantum coherent capacitor the $\mathrm{RC}$ time can still be written as the product of a resistance and a capacitance, i.e. $\tau_{R C}=R_{q} C_{\mu}$. However, due to the coherent nature of electron transport through mesoscopic structures, both the electrochemical capacitance $C_{\mu}$, which determines the charge on the capacitor and the charge relaxation resistance $R_{q}$, which governs the charge fluctuations, now crucially depend on coherence properties of the system. The capacitance $C_{\mu}$ is related to the imaginary part of the $\mathrm{AC}$ conductance but it can also be obtained by the differentiation of a thermodynamic (grand-canonical) potential [2, 3, 4, 5, 6]. The charge relaxation resistance $R_{q}$ is related to the real part of the $\mathrm{AC}$ conductance and therefore requires a dynamic theory. In analogy to the classical RC circuit depicted in the upper left corner of Fig. [1] one has for the mesoscopic system

$$
G(\omega)=-i \omega C_{\mu}+\omega^{2} C_{\mu}^{2} R_{q}+O\left(\omega^{3}\right) .
$$

This equation will be taken as a definition of $C_{\mu}$ and $R_{q}$. If the cavity-reservoir connection permits the transmission of only a single spin polarized channel, a selfconsistent scattering matrix approach, gives at zero temperature a resistance equal to half a resistance quantum [1]

$$
R_{q}=\frac{h}{2 e^{2}}
$$

We emphasize that the factor of 2 is not connected to spin but is rather due to the fact that the cavity connects to only one electron reservoir. More astonishing, even counter-intuitive, is the fact that Eq. (2) is independent of the transmission properties of the channel.

In a seminal experiment, J. Gabelli et al. 7] have recently measured both the in and out of phase parts of the $\mathrm{AC}$ conductance of a mesoscopic RC circuit. In their experiment one "plate" of the capacitor consists of a submicrometer quantum dot (QD) and the other is formed by a macroscopic top gate. The role of the resistor is played by a tunable quantum point contact (QPC) connecting the QD to an electron reservoir. The results of this experiment are in good agreement with the theoretical predictions of [1], in particular they confirm the universality of the single channel charge relaxation resistance by using a strong magnetic field to spin polarize the electrons. However, it is a priori unclear whether the results derived in Ref. 11 still hold in the presence of single charge effects [2, 3, 44 which must become important if the transmission through the QPC becomes small. Indeed the experiment observes Coulomb blockade oscillations of the capacitance as a function of the gate voltage. It is the aim of the present work to present a theoretical description for the charge relaxation resistance in the presence of Coulomb blockade effects.

The mesoscopic RC circuit along with the principal

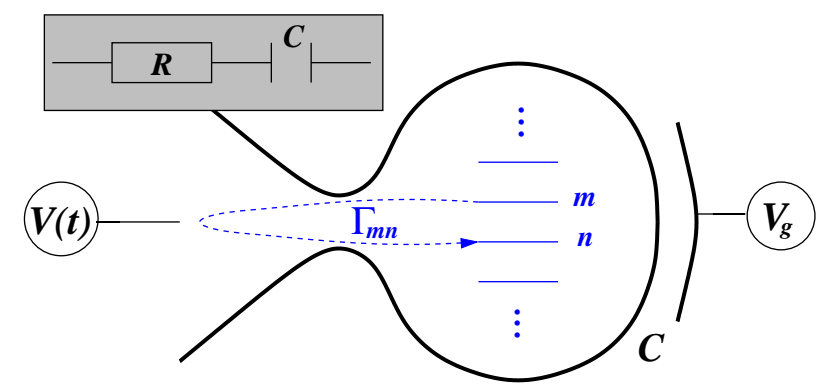

FIG. 1: Schematics of the mesoscopic capacitor. A cavity is connected via one lead to an electron reservoir at voltage $V(t)$ and capacitively coupled to a backgate with voltage $V_{g}$. The coupling matrix elements $\Gamma_{m n}$ are defined in the text. The inset shows the corresponding classical RC circuit. 
model parameters is shown in Fig. 1. $V(t)=V_{\mathrm{ac}} \cos (\omega t)$ is the time dependent voltage applied to the electron reservoir while $V_{g}$ is the voltage applied to the gate and $C$ is the geometrical capacitance between the $\mathrm{QD}$ and the gate. The matrix elements $\Gamma_{m n}$, to be defined below, describe the indirect coupling between the dot states $m$ and $n$ via the reservoir.

Our first goal is to show that in the single channel case $R_{q}$ is universal also in the Coulomb blockade regime. For that purpose we treat the cavity at the Hartree-Fock (HF) level [8, 9]. The starting point of our calculation is the relation

$$
I(t)=-e \frac{\partial}{\partial t} \Im\left\{\operatorname{tr}\left[G^{<}(t, t)\right]\right\},
$$

which expresses the tunneling current through the QPC as a function of the lesser (matrix) Green function (GF) $G^{<}\left(t, t^{\prime}\right)$ of the dot. We are interested in the regime where the modulation energy is small compared to the level spacing in the dot. Treating the dot as zero dimensional, the Hamiltonian of this system is [10]

$$
H=H_{L}+H_{D}+\sum_{k \sigma, n}\left(t_{k, n}^{\sigma} c_{k \sigma}^{\dagger} d_{n \sigma}+\text { h.c. }\right)
$$

where $H_{L}=\sum_{k \sigma} E_{k \sigma}(t) c_{k \sigma}^{\dagger} c_{k \sigma}$, with $E_{k \sigma}(t)=E_{k \sigma}^{0}+$ $e V(t)$, describes the (non-interacting) electrons in the isolated lead and $t_{k, n}^{\sigma}$ is the tunneling matrix element between a reservoir momentum state $k$ and the $n$-th single particle dot state, both with spin $\sigma$. A change in the gate voltage is modeled as a shift of the Fermi energy $E_{F}$ in the reservoir and we set $V_{g}=0$. The Hamiltonian of the dot reads $H_{D}=\sum_{n \sigma} \epsilon_{n \sigma} d_{n \sigma}^{\dagger} d_{n \sigma}+E_{c}\left(\hat{N}_{d o t}+\mathcal{N}(t)\right)^{2}$. Here $E_{c}=e^{2} / 2 C$ is the electrostatic charging energy, $\hat{N}_{d o t}=$ $\sum_{m \sigma} d_{m \sigma}^{\dagger} d_{m \sigma}$ is the particle number in the dot and $e \mathcal{N}(t)=C U(t)$ gives the polarization charges between dot and gate produced by the time dependent voltage at the reservoir 11]. This polarization charge in turn, leads to a time-dependent (Hartree) potential $U(t)$ inside the dot and we may write $H_{D}=\sum_{n \sigma} \tilde{\epsilon}_{n \sigma}(t) d_{n \sigma}^{\dagger} d_{n \sigma}+E_{c} \hat{N}_{d o t}^{2}$, with $\tilde{\epsilon}_{m \sigma}(t)=\epsilon_{m \sigma}+e U(t)$. In $\mathrm{HF}$ approximation, the retarded (advanced) GF of the dot takes the form [12, 13.

$$
G^{R(A)}\left(t, t^{\prime}\right)=\mathrm{e}^{i \phi_{U}\left(t, t^{\prime}\right)} G_{e q}^{R(A)}\left(t-t^{\prime}\right),
$$

where $G_{e q}^{R(A)}\left(t-t^{\prime}\right)=\left.G^{R(A)}\left(t, t^{\prime}\right)\right|_{V_{\mathrm{ac}}=0}$ is the equilibrium retarded (advanced) HF Green function and $\phi_{U}\left(t, t^{\prime}\right)=$ $\int_{t}^{t^{\prime}} d \tau U(\tau)$. The equal time lesser GF is obtained via the Keldysh equation 12, 14]

$$
G^{<}(t, t)=\int d t_{1} \int d t_{2} G^{R}\left(t, t_{1}\right) \Gamma^{<}\left(t_{1}, t_{2}\right) G^{A}\left(t_{2}, t\right) .
$$

Here $\Gamma^{<}\left(t, t^{\prime}\right)=i \Gamma \mathrm{e}^{i \phi_{V}\left(t, t^{\prime}\right)} \hat{f}\left(t-t^{\prime}\right)$ with $\phi_{V}\left(t, t^{\prime}\right)=$ $\int_{t}^{t^{\prime}} d \tau V(\tau)$ is the lesser coupling self-energy and $\hat{f}\left(t-t^{\prime}\right)$
$=(1 / 2 \pi) \int d E \mathrm{e}^{-i E\left(t-t^{\prime}\right)} f(E)$, is the Fourier transform of the Fermi function. $\Gamma_{m n}^{\sigma}=2 \pi \rho_{L} t_{m}^{\sigma}{ }^{*} t_{n}^{\sigma}$ are the coupling matrix elements in the wide band limit [12]. Here and in the following, we use the matrix notation $A_{m \sigma, n \sigma^{\prime}} \equiv$ $A_{m n}^{\sigma} \delta_{\sigma \sigma^{\prime}}$, which takes advantage of the fact that spin is conserved in (4). An important property of the coupling matrix elements is that $\Gamma_{m n}^{\sigma} \Gamma_{k l}^{\sigma}=\Gamma_{m l}^{\sigma} \Gamma_{k n}^{\sigma}$, from which it immediately follows that for arbitrary matrices $A$ and $B$

$$
\operatorname{tr}\left[\Gamma^{\sigma} A \Gamma^{\sigma} B\right]=\operatorname{tr}\left[\Gamma^{\sigma} A\right] \operatorname{tr}\left[\Gamma^{\sigma} B\right]
$$

where the trace is over a basis of dot states with spin $\sigma$. Since we are interested in the linear conductance, we expand (6) to linear order in $V$ and $U$ and find after double Fourier transformation

$$
G^{<}\left(E, E^{\prime}\right)=G_{e q}^{R}(E) \Gamma_{1}^{<}\left(E, E^{\prime}\right) G_{e q}^{A}\left(E^{\prime}\right)+O\left(U^{2}, V^{2}\right),
$$

where $\Gamma_{1}^{<}\left(E, E^{\prime}\right)$ is the double Fourier transform of $\Gamma_{1}^{<}\left(t, t^{\prime}\right)=i \Gamma\left(1+i \phi\left(t, t^{\prime}\right)\right) \hat{f}\left(t-t^{\prime}\right)$ with $\phi\left(t, t^{\prime}\right) \equiv$ $\phi_{V}\left(t, t^{\prime}\right)-\phi_{U}\left(t, t^{\prime}\right)$. With (3) and (8) the linear response tunneling current at frequency $\omega$ becomes $I(\omega)=$ $g(\omega)[V(\omega)-U(\omega)]$, with

$$
g(\omega)=-i \frac{e \omega}{2 \pi} \int d E F(E, \omega) \operatorname{tr}\left[G_{e q}^{R}(E) \Gamma G_{e q}^{A}(E-\omega)\right]
$$

where $F(E, \omega)=[f(E+\omega)-f(E)] / \omega$ and we have set $\hbar=$ 1. To obtain the AC conductance $G(\omega)=I(\omega) / V(\omega)$, we need the internal potential $U(\omega)$. For this we note that in the present single lead system, the displacement current $-i \omega e \mathcal{N}(\omega)$ is equal to the tunneling current so that $g(\omega)[V(\omega)-U(\omega)]=-i \omega C U(\omega)$ and consequently [15] $U(\omega)=g(\omega) V(\omega) /[-i \omega C+g(\omega)]$. Expanding the conductance to second order in frequency, we then obtain after restoring the units

$$
R_{q}=-\frac{h}{2 e^{2}} \frac{\int d E f^{\prime}(E) \operatorname{tr}\left[D(E)^{2}\right]}{\left(\int d E f^{\prime}(E) \operatorname{tr}[D(E)]\right)^{2}}
$$

where $f^{\prime}=d f / d E$ and $D(E) \equiv G_{e q}^{R}(E) \Gamma G_{e q}^{A}(E)$. Using (7), we have $\operatorname{tr}\left[D^{\sigma}(E)^{2}\right]=\operatorname{tr}\left[D^{\sigma}(E)\right]^{2}$ and hence at zero temperature

$$
R_{q}=\frac{h}{2 e^{2}} \frac{\sum_{\sigma} \nu_{\sigma}\left(E_{F}\right)^{2}}{\left(\sum_{\sigma} \nu_{\sigma}\left(E_{F}\right)\right)^{2}}
$$

where $\nu_{\sigma}(E) \equiv \operatorname{tr}\left[D^{\sigma}(E)\right] / 2 \pi$ is the density of spin $\sigma$ states in the dot. Eqs. 911) are central results of this work. In particular, Eq. (11) demonstrates that for a single (spin polarized) channel $R_{q}$ is still given by Eq. (2).

In the following, we use Eq. (11) to investigate the magnetic field dependence of $R_{q}$. We consider here a dot with two spin degenerate levels with bare energies $\epsilon_{1 \sigma}$ and $\epsilon_{2 \sigma}=\epsilon_{1 \sigma}+\Delta$ respectively. In the numerical calculations, we set $E_{c} / \Delta=2.5$. We are interested 


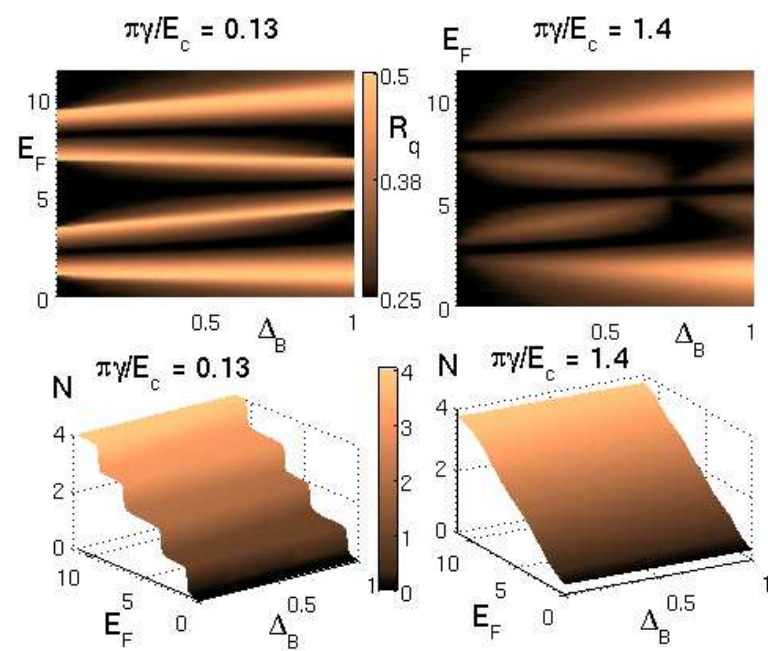

FIG. 2: Magnetic field dependence of the charge relaxation resistance $R_{q}$. The upper panels show $R_{q}$ as a function of the Zeeman splitting $\Delta_{B}$ and the Fermi energy $E_{F}$ for weak $\pi \gamma / E_{c}=0.13$ and strong $\pi \gamma / E_{c}=1.4$ coupling. All energies are given in units of the bare level spacing $\Delta$. The lower panels show the corresponding total dot charge.

in the regime of low magnetic field, where the Zeeman splitting $\Delta_{B}=\mu_{B} g B \leq \Delta$. For simplicity, we further assume that $\Gamma_{m n}^{\uparrow}=\Gamma_{m n}^{\downarrow} \equiv \gamma$, for $m, n \in\{1,2\}$. The equilibrium HF retarded GF of the dot may be written as $G_{e q}^{R}(E)=\left[G_{0}^{R}(E)^{-1}-\Sigma_{H F}^{R}+i \Gamma / 2\right]^{-1}$, with the non-interacting equilibrium GF of the isolated dot $\left(G_{0}^{R}(E)\right)_{m n}=\delta_{m n}\left(E-\epsilon_{m}+i 0^{+}\right)^{-1}$ and the HF selfenergy

$$
\left(\Sigma_{H F}^{R}\right)_{m n}^{\sigma}=E_{c}\left[\delta_{m n} \sum_{l \sigma^{\prime}}\left\langle n_{l \sigma^{\prime}}\right\rangle-\left\langle d_{m \sigma}^{\dagger} d_{n \sigma}\right\rangle\right]
$$

The most important feature of this self-energy is that it correctly excludes the unphysical self-interaction terms ( $m=n=l$ and $\sigma=\sigma^{\prime}$ ) of the Hartree approximation and consequently leads to the appearance of the Coulomb gap across $E_{F}$, which is the essential spectral signature of the Coulomb blockade effect. The "mean fields" $\left\langle d_{m \sigma}^{\dagger} d_{n \sigma}\right\rangle$ are determined self-consistently [16] by solving the set of equations

$$
\left\langle d_{m \sigma}^{\dagger} d_{n \sigma}\right\rangle=\frac{1}{2 \pi} \int_{-\infty}^{\infty} d E f(E)\left[G_{e q}^{R}(E) \Gamma G_{e q}^{A}(E)\right]_{m n}^{\sigma} .
$$

Because of the interaction, $\nu_{\sigma}(E)$ depends on the level occupancies and we must distinguish two cases. Solving Eq. (13) numerically in the strongly blockaded regime $\pi \gamma / E_{c} \ll 1$, we find that $R_{q}$ is non-universal even as $B \rightarrow 0$ (upper left panel of Fig. 22). This is due to the fact that the dot charge is strongly quantized in this regime, as shown in the lower left panel of Fig. 2 2 which leads to a gap of order $E_{c}+\Delta \delta_{\sigma \sigma^{\prime}}+\left(1-\delta_{\sigma \sigma^{\prime}}\right) \Delta_{B}$ between the highest occupied state with spin $\sigma$ and the lowest unoccupied state with spin $\sigma^{\prime}$. There are thus four well separated (separation $\sim E_{c}$ ), narrow (width $\sim \gamma$ ) resonances in the total density of states $\sum_{\sigma} \nu_{\sigma}$ as a function of $E_{F}$. We can understand the particular behavior of $R_{q}$ shown in the upper left panel of Fig. 2 at specific values of the Fermi energy. If $E_{F}$ is on a resonance with spin $\sigma$, then $\nu_{\sigma^{\prime}}\left(E_{F}\right) \cong \nu_{\sigma}\left(E_{F}\right) \delta_{\sigma \sigma^{\prime}}$. Therefore on resonance, i.e. for $E_{F} \in\left\{\epsilon_{1 \sigma}, \epsilon_{2 \sigma}, \epsilon_{1 \bar{\sigma}}+E_{c}+\Delta_{B}, \epsilon_{2 \bar{\sigma}}+E_{c}+\Delta_{B}\right\}$, we expect to have $R_{q} \cong h / 2 e^{2}$ according to (11). Furthermore, in the middle between two consecutive resonances $\nu_{\sigma}\left(E_{F}\right)=\nu_{\bar{\sigma}}\left(E_{F}\right)$ and so $R_{q}$ takes on its minimal two channel value $h / 4 e^{2}$. In the opposite limit of strong coupling $1 \lesssim \pi \gamma / E_{c}$, the Coulomb blockade is smeared out and the charge on the dot is not strongly quantized anymore (see lower right panel in Fig. 2). Thus at very low magnetic fields, the two spin states are nearly simultaneously charged and the degeneracy is not appreciably lifted. Therefore, $R_{q} \cong h / 4 e^{2}$ at low magnetic fields as shown in the upper right panel of Fig. 20 As $\Delta_{B}$ increases the broad resonances in the density of states corresponding to the two spin degenerate levels split into consecutively overlapping resonances and $R_{q}$ starts to increases except in the middle between two neighboring resonances. Finally, a crossing of the two innermost resonances is observed for $\Delta_{B} \cong 0.75$. Such a crossing occurs when the Zeeman splitting approaches the effective level spacing $\Delta+E_{c}\left(\left\langle n_{1 \sigma}\right\rangle-\left\langle n_{2 \bar{\sigma}}\right\rangle\right)$, which interestingly is seen to be smaller than the bare level spacing here. This effect is peculiar to the strongly coupled regime and is due to enhanced exchange interactions, which favor the consequent population of states with equal spins [17, 18]. At the crossing point the densities of both spin states are equal and $R_{q}$ takes on its minimal value.

As a second application, we investigate the temperature dependence of $R_{q}$ in the high magnetic field limit, where the incoming electrons are effectively spin polarized and there is only a single transmitting channel through the QPC. We consider here a quantum dot with two (bare) levels $\epsilon_{1}$ and $\epsilon_{2}=\epsilon_{1}+\Delta$ and suppress the now superfluous spin index. In the low temperature regime $k_{B} T \ll \gamma$, we may expand $\nu(E)$ and $\nu(E)^{2}$ around $E_{F}$ assuming that these functions vary slowly in the range $E_{F} \pm k_{B} T / 2$. This yields, to first non-vanishing order in $k_{B} T \equiv \beta^{-1}$

$$
R_{q} \cong \frac{h}{2 e^{2}}\left(1+\frac{\pi^{2}}{3 \beta^{2}}\left[\frac{\nu^{\prime}\left(E_{F}\right)}{\nu\left(E_{F}\right)}\right]^{2}\right),
$$

where $\nu^{\prime}(E) \equiv \frac{\partial \nu(E)}{\partial E}$. Thus in HF approximation, the lowest order correction is proportional to the square of the energy derivative of the density of states at the Fermi energy. This explains the presence of the peaks seen in Fig. 3 to the left and right of the two resonances at $E_{F}=\epsilon_{1}=1$ and $E_{F}=\epsilon_{2}^{*}=\epsilon_{1}+E_{c}+\Delta$, for the two lowest temperatures $\beta=100$ and $\beta=12.5$. The fact that for $\beta=12.5, R_{q}$ does not identically vanish at resonance 


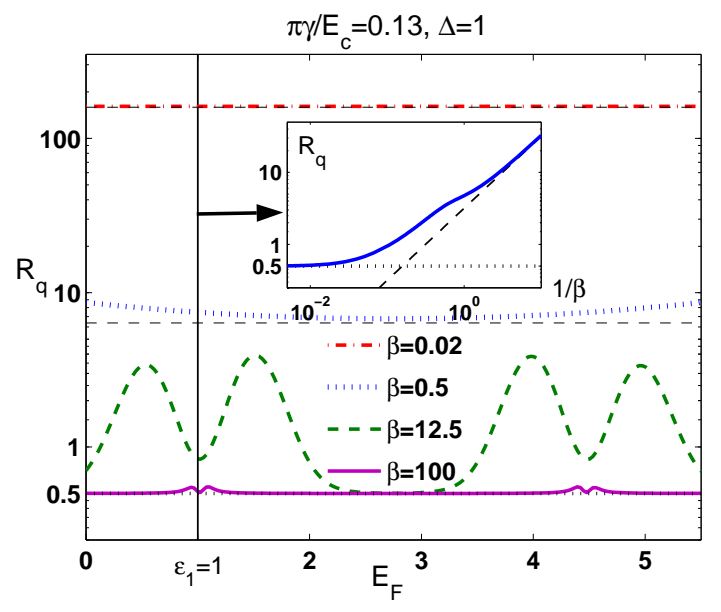

FIG. 3: Charge relaxation resistance $R_{q}$ as a function of Fermi energy $E_{F}$ for different temperatures. The lower three curves are for a two level dot. The upper most curve is the high temperature asymptote. The inset shows $R_{q}$ as a funtion of temperature for $E_{F}=\epsilon_{1}$.

where $\nu^{\prime}\left(E_{F}\right)=0$, is due to higher order terms in the low temperature expansion, which involve non-vanishing higher order derivatives of $\nu$. The dotted horizontal line at $R_{q}=h / 2 e^{2}$ marks the zero temperature result. In the opposite limit of very high temperature $k_{B} T \gg \Delta+E_{c}$, where $-f^{\prime}(E) \cong \beta / 4$ and in the weakly coupled regime $\gamma \ll \Delta$, where the density of states is well approximated by a sum of displaced Lorentzians of width $\gamma$, we can estimate the remaining integrals in the numerator and denominator of (10) as $-\int d E f^{\prime}(E) \nu(E)^{2} \cong \beta M / 4 \pi \gamma$ and $-\int d E f^{\prime}(E) \nu(E) \cong \beta M / 4$ respectively, where $M$ is the number of density of states peaks under the broad curve $f^{\prime}(E)$. Therefore at very high temperature we find

$$
R_{q} \cong \frac{h}{e^{2}} \frac{2}{\pi \gamma M \beta}
$$

This high temperature limit is shown as a dashed horizontal black line in Fig. 3 for the two highest temperatures. In the present two level system, $M=2$ and as expected the agreement with the numerical integration of (10) is good for the highest temperature $\beta^{-1}=50 \gg \Delta+E_{c}=3.5$. For the intermediate temperature $\Delta=1<\beta^{-1}=2<\Delta+E_{c}=3.5$, there is already a significant deviation from the asymptotic result. In the inset of Fig. [3 we show $R_{q}$ as a function of the temperature on the first resonance at $E_{F}=\epsilon_{1}$. The dashed line corresponds to the high temperature asymptote (15) and the dotted line is the low temperature limit (14).

In this work we have analyzed the charge relaxation of a mesoscopic capacitor in the linear regime of coherent dynamical transport. We have shown that the single channel zero temperature charge relaxation resistance $R_{q}$ is universal even in the presence of single charge effects, described in the Hartree-Fock approximation. This shows in particular that charge relaxation of a quantum coherent capacitor is faster than one could naively expect based on classical arguments. We obtain the magnetic field dependence of $R_{q}$ in the two channel case (electrons with spin), where we identify two qualitatively different regimes of weak and strong coupling. In the former, the degeneracy of both spin states is lifted by the interaction at all field strengths and $R_{q}$ is non-universal. In the latter regime, the degeneracy is lifted only at finite field and at zero field $R_{q}$ is universal and equal to its minimum two channel value $h / 4 e^{2}$. The finite temperature behavior of $R_{q}$ for a two level spin polarized system is, to lowest order, determined by the logarithmic derivative of the density of states with respect to energy. In the multilevel case the HF approximation gives a reasonable qualitative picture of the underlying physics. The important case of $B=0$, for a single strongly coupled level in the dot, requires a treatment of Kondo physics and will be discussed elsewhere 19.

Our work demonstrates that mesoscopic charge relaxation is a physically very interesting process and provides a basis for the understanding of experimental data in the low and high magnetic field ranges.

We thank C. Glattli and J. Gabelli for discussions and sharing their data with us. This work was supported by the Swiss NSF, MaNEP, the Spanish MEC by MAT200507369-C03-03 and the Ramon y Cajal program.

[1] M. Büttiker, H. Thomas, and A. Prêtre, Phys. Lett. A 180, 364 (1993).

[2] K. Flensberg, Phys. Rev. B 48, 11156 (1993).

[3] K. A. Matveev, Phys. Rev. B 51, 1743 (1995); K. Le Hur and G. Seelig, Phys. Rev. B 65, 165338 (2002).

[4] I. L. Aleiner, P. W. Brouwer and L. I. Glazman, Phys. Rep. 358, 309 (2002).

[5] M. Büttiker and C. A. Stafford, Phys. Rev. Lett. 76, 495 (1996).

[6] T. Duty et al., Phys. Rev. Lett. 95, 206806 (2005); M. A. Sillanpää et al., ibid. 95, 206806 (2005).

[7] J. Gabelli, J. M. Berroir, G. Fève, B. Plaçais, Y. Jin, B. Etienne and D. C. Glattli, Science (unpublished); J. Gabelli, PhD dissertation, ENS Paris 6 (2006).

[8] P. M. Brouwer, A. Lamacraft and K. Flensberg, Phys. Rev. B 72, 075316 (2005).

[9] G. Hackenbroich, Phys. Rep. 343, 463 (2001).

[10] G. Platero and R. Aguado, Phys. Rep. 395, 1 (2004).

[11] Ph. Brune, C. Bruder and H. Schoeller, Phys. Rev. B 56, 4730 (1997).

[12] A. P. Jauho, N. S. Wingreen and Y. Meir, Phys. Rev. B 50, 5528 (1994).

[13] R. López et al., Phys. Rev. B 64, 075319 (2001).

[14] B. Wang and H. Guo, Phys. Rev. Lett. 82, 398 (1999).

[15] A. Prêtre, H. Thomas and M. Büttiker, Phys. Rev. B 54, 8130 (1996).

[16] D. I. Golosov and Y. Gefen, cond-mat/0601342

[17] L. P. Rokhinson et al., Phys. Rev. B, 63, 035321 (2001).

[18] I. L. Kurland, R. Berkovits and B. L. Altshuler, Phys. 
Rev. Lett. 86, 3380 (2001).

[19] R. López, S. E. Nigg and M. Büttiker (unpublished). 